\title{
Robust anti-windup control for marine cyber-physical systems
}

\author{
Mikhail Kakanov, Mikhail Kaliuzhny, Oleg Borisov, Vladislav Gromov, Sergey Vlasov, Sergey Somov and Anton Pyrkin \\ ITMO University, Department of Computer Science and Control Systems, 19710149 Kronverksky Pr., St. Petersburg, Russia
}

\begin{abstract}
In this paper the robust output control with anti-windup compensation and its implementation to the robotic boat are addressed. The detailed control design and stability analysis of the closed-loop systems are provided in the work. Extensive experimental verification of the dynamic positioning system based on various modifications of the basic controller is carried out by means of robotic boat. The corresponding experimental results are presented and analysed.
\end{abstract}

\section{Introduction}

Development of cyber-physical systems (CPS) is a brand new ongoing scientific trend in applied control theory. It assumes design of sophisticated systems comprised of multiple smart devices interconnected between each other and able to make decisions on their own and adapt to external changes without human intervention. There is a wide range of applications of such systems including for instance manufacturing and transportation.

This research is focused on development of robust output control algorithm with anti-windup compensation for MIMO plants with uncertain parameters and bounded inputs. In particular a solution suggested in this paper can be used in dynamic positioning (DP) problem to control a surface vessel being a component of marine CPS.

If an integral term being a part of the internal model is included in the control system, input saturation might cause various adverse effects when implementing regulator to real plants. Anti-windup modification first mentioned in [1] can be used to resolve this issue. There is a number of scientific works developing anti-windup approaches. Overview of modern anti-windup design is consider in [2]. Application of the toolkit of linear matrix inequalities (LMI) to synthesis is presented in [3-8]. The anti-windup is combined with internal control mode (ICM) in [9-11]. In the application to discrete-time systems, the anti-windup scheme is considered in [12]. The consecutive compensator approach described in [13, 14] and based on the pacification approach [15] is used as a basis for control design for marine CPS. The relevant problem of output regulation for MIMO systems with unknown disturbance compensation and application of the suggested control approach to surface vessels to control its velocities is addressed in [16]. Detailed theoretical background on ship control is provided in [17]. Some results on saturated control design being applied to the research setup of the robotic boat is presented [18, 19] and to the quadcopters in [20-22].

The consecutive compensator approach is augmented with an auxiliary integral loop and anti-windup scheme. Such solution is needed, on the one hand, to eliminate the steady state error and, on the other hand, to compensate integral windup.

This paper is organized as follows. The problem of this research is formulated in Section 2. Control design is given in Section 3. Stability analysis of the closed-loop system is provided in Section 4. Experimental verification and the corresponding test results are described and discussed in Section 5. The paper is summarized in Conclusions.

\section{Problem formulation}

Consider the plant

$$
\begin{gathered}
\dot{x}=A \boldsymbol{x}+\boldsymbol{b} u+R \boldsymbol{\omega}, \\
y=\boldsymbol{c}^{\boldsymbol{T}} \boldsymbol{x},
\end{gathered}
$$

where $\boldsymbol{x} \in \mathbb{R}^{n}$ is the state, $\boldsymbol{\omega} \in \mathbb{R}^{n_{\omega}}$ is the disturbance, which derivative and initial conditions are

$$
\dot{\omega}=0, \quad \omega(0) \neq 0,
$$

$y \in \mathbb{R}^{1}$ is the measurable output, $u \in \mathbb{R}^{1}$ is the input satisfying

$$
u=\operatorname{sat}(v)=\left\{\begin{array}{c}
u_{\max } \text { if } v \geq u_{\text {max }} \\
u \text { if } u_{\min }<v<u_{\max } \\
u_{\min } \text { if } v \leq u_{\min }
\end{array}\right.
$$

where $u_{\max }$ and $u_{\min }$ are the input saturation limits, $v$ is the control signal generated by the nominal linear controller, $A, R, \boldsymbol{b}, \boldsymbol{c}$ are matrices and vectors of corresponding dimensions.

Assumption 1: The plant (1), (2) is minimum phase.

Assumption 2: The relative degree of the plant (1), (2) $\rho \geq 1$ is known.

Assumption 3: The input saturation limits $u_{\max }$ and $u_{\text {min }}$ have different signs, but the same magnitude.

Assumption 4: The disturbance is bounded $\omega \in \mathcal{L}_{\infty}$ and the nominal control signal $u_{0}$ needed for its compensation at steady state satisfies

\footnotetext{
Corresponding author: kakanov.mikhail@niuitmo.ru
} 


$$
u_{\min } \leq\left|u_{0}\right| \leq u_{\max } .
$$

The objective is to asymptotically stabilize the output under conditions of external disturbance effects and presence of the integral windup.

\section{Robust control design}

The plant model (1), (2) can be represented as

$$
y(s)=\frac{b(s)}{a(s)} \bar{u}(s)+\frac{r(s)}{a(s)} \omega(s),
$$

where $b(s)$ is the Hurwitz polynomial due to Assumption 1.

Rewrite the plant model (5) as

$$
\begin{gathered}
\frac{a(s)}{b(s)} y(s)=\bar{u}(s)+\frac{r(s)}{b(s)} \omega(s), \\
\left(c(s)+\frac{d(s)}{b(s)}\right) y(s)=\bar{u}(s)+\left(r_{2}(s)+\frac{r_{1}(s)}{b(s)}\right) \omega(s), \\
c(s) y(s)=\left(\bar{u}(s)-\frac{d(s)}{b(s)} y(s)+\frac{r_{1}(s)}{b(s)} \omega(s)\right) \\
+r_{2}(s) \omega(s) .
\end{gathered}
$$

and express (6) as two interconnectyed subsystems as

$$
\begin{gathered}
z(s)=\frac{d(s)}{b(s)} y(s)-\frac{r_{1}(s)}{b(s)}, \\
y(s)=\frac{1}{c(s)}(\bar{u}(s)-z(s))+\frac{r_{2}(s)}{c(s)} \omega(s),
\end{gathered}
$$

which is equivalent to the state-space model

$$
\begin{gathered}
{\left[\begin{array}{l}
\dot{z_{1}} \\
\dot{z_{2}}
\end{array}\right]=\left[\begin{array}{ll}
A_{11} & A_{12} \\
A_{21} & A_{22}
\end{array}\right]\left[\begin{array}{l}
Z_{1} \\
z_{2}
\end{array}\right]+\left[\begin{array}{c}
0 \\
b_{2}
\end{array}\right] \bar{u}+\left[\begin{array}{l}
R_{1} \\
R_{2}
\end{array}\right] \omega,} \\
y=\left[\begin{array}{ll}
0 & c^{T}
\end{array}\right]\left[\begin{array}{l}
Z_{1} \\
z_{2}
\end{array}\right],
\end{gathered}
$$

where $A_{11}$ is the Hurwitz matrix due to Assumption 1, if the matrix $A_{22}$ is chosen in companion form, then $\boldsymbol{b}_{2}^{T}=\left[\begin{array}{llll}0 & \cdots & 0 & b_{0}\end{array}\right], \boldsymbol{c}_{2}^{T}=\left[\begin{array}{llll}1 & 0 & \cdots & 0\end{array}\right]$.

Choose the control law

$$
\begin{array}{r}
v=-\kappa\left(c_{q}^{T} \xi+y\right)-\gamma \eta, \\
\dot{\xi}=A_{q} \xi+b_{q} y, \\
\dot{\eta}=\kappa\left(c_{q}^{T} \xi+y\right)+v \psi(v), \\
\psi(v)=v-\operatorname{sat}(v),
\end{array}
$$

where $\psi(v)$ is the memoryless nonlinearity, $\kappa>0$, $\gamma>0, v>0, A_{q}, \boldsymbol{b}_{\boldsymbol{q}}, c_{q}$ are given as

$$
\begin{gathered}
A_{q}=\left[\begin{array}{ccccc}
q_{\rho}^{\prime} \sigma & 1 & 0 & \cdots & 0 \\
-q_{\rho-1}^{\prime} \sigma^{2} & 0 & 1 & \cdots & 0 \\
\vdots & \vdots & \vdots & \ddots & \vdots \\
-q_{2}^{\prime} \sigma^{\rho-1} & 0 & 0 & \cdots & 1 \\
-q_{1}^{\prime} \sigma^{\rho} & 0 & 0 & \cdots & 0
\end{array}\right], \\
b_{q}^{T}=\left[\begin{array}{lllll}
q_{\rho}^{\prime} \sigma & q_{\rho-1}^{\prime} \sigma^{2} & \cdots & q_{2}^{\prime} \sigma^{\rho-1} & q_{1}^{\prime} \sigma^{\wedge} \rho
\end{array}\right], \\
c_{q}^{T}=\left[\begin{array}{lllll}
q_{1} & q_{2} & \cdots & q_{\rho-1} & q_{\rho}
\end{array}\right],
\end{gathered}
$$

where $\sigma>0$ and $q_{i}^{\prime}(i=\overline{1, \rho})$ are chosen for the system (10) to be Hurwitz, $q_{i}(i=\overline{1, \rho})$ are coefficients of an arbitrary polynomial of the degree $\rho-1$.

Interconnecting the plant (7), (8) and the control law (9)-(12) obtain the model of the closed-loop system (13).

Perform the change of coordinates $\chi=z_{2}-\xi$ and compute the derivative

$$
\begin{aligned}
\dot{\chi}=\dot{z}_{2}-\dot{\xi}=A_{21} & z_{1}+\left(A_{22}-I_{0}-\kappa b_{2}\left(c_{q}^{T}+c_{2}^{T}\right)\right) z_{2} \\
& +\left(A_{q}+\kappa b_{2} c_{q}^{T}\right) \chi-b_{2} \gamma \eta-b_{2} \psi \\
& +R_{2} \omega
\end{aligned}
$$

where

$$
I_{0}=A_{q}+b_{q} c_{2}^{T}=\left[\begin{array}{ccccc}
0 & 1 & 0 & \ldots & 0 \\
0 & 0 & 1 & \ldots & 0 \\
\vdots & \vdots & \vdots & \ddots & \vdots \\
0 & 0 & 0 & \ldots & 0
\end{array}\right]
$$

This change of coordinates puts the system (13) into the form (14).

Perform the second change of variables $\zeta=$ $\left(\boldsymbol{b}_{2}^{T} \boldsymbol{b}_{2}\right)^{-1} \boldsymbol{b}_{2}^{T} z_{2}+\eta$ and compute the derivative

$$
\begin{aligned}
\dot{\zeta}=\left(\boldsymbol{b}_{2}^{T} \boldsymbol{b}_{2}\right)^{-1} \boldsymbol{b}_{2}^{T} \dot{z}_{2} & +\dot{\eta} \\
& =\left(\boldsymbol{b}_{2}^{T} \boldsymbol{b}_{2}\right)^{-1} \boldsymbol{b}_{2}^{T} A_{21} z_{1} \\
& +\left(\boldsymbol{b}_{2}^{T} \boldsymbol{b}_{2}\right)^{-1} \boldsymbol{b}_{2}^{T}\left(A_{22}+\gamma I\right) z_{2}-\gamma \zeta \\
& +(v-1) \psi+\left(\boldsymbol{b}_{2}^{T} \boldsymbol{b}_{2}\right)^{-1} \boldsymbol{b}_{2}^{T} R_{2} \boldsymbol{\omega},
\end{aligned}
$$

where $I$ is the identity matrix of the corresponding dimension.

For the sake of simplicity denote

$$
\bar{b}_{2}^{T}=\left(\boldsymbol{b}_{2}^{T} \boldsymbol{b}_{2}\right)^{-1} \boldsymbol{b}_{2}^{T} .
$$

This change of coordinates taking into accound (15) puts the system (14) into the form (16) : 


$$
\begin{aligned}
& {\left[\begin{array}{c}
\dot{Z}_{1} \\
\dot{z}_{2} \\
\dot{\xi} \\
\dot{\eta}
\end{array}\right]=\left[\begin{array}{cccc}
A_{11} & A_{12} & 0 & 0 \\
A_{21} & A_{22}-\kappa \boldsymbol{b}_{2} \boldsymbol{c}_{2}^{\boldsymbol{T}} & -\kappa \boldsymbol{b}_{2} \boldsymbol{c}_{\boldsymbol{q}}^{\boldsymbol{T}} & -\boldsymbol{b}_{2} \gamma \\
0 & \boldsymbol{b}_{\boldsymbol{q}} \boldsymbol{c}_{2}^{\boldsymbol{T}} & A_{q} & 0 \\
0 & \kappa \boldsymbol{c}_{2}^{T} & \kappa \boldsymbol{c}_{\boldsymbol{q}}^{\boldsymbol{T}} & 0
\end{array}\right]\left[\begin{array}{c}
z_{1} \\
z_{2} \\
\xi \\
\eta
\end{array}\right]+\left[\begin{array}{c}
0 \\
-\boldsymbol{b}_{2} \\
0 \\
v
\end{array}\right] \psi+\left[\begin{array}{c}
R_{1} \\
R_{2} \\
0 \\
0
\end{array}\right] \omega} \\
& {\left[\begin{array}{c}
\dot{z}_{1} \\
\dot{z}_{2} \\
\dot{\chi} \\
\dot{\eta}
\end{array}\right]=\left[\begin{array}{cccc}
A_{11} & A_{12} & 0 & 0 \\
A_{21} & A_{22}-\kappa \boldsymbol{b}_{2}\left(\boldsymbol{c}_{\boldsymbol{q}}^{\boldsymbol{T}}+\boldsymbol{c}_{2}^{\boldsymbol{T}}\right) & -\kappa \boldsymbol{b}_{2} \boldsymbol{c}_{\boldsymbol{q}}^{\boldsymbol{T}} & -\boldsymbol{b}_{2} \gamma \\
A_{21} & A_{22}+I_{0}-\kappa \boldsymbol{b}_{2}\left(\boldsymbol{c}_{\boldsymbol{q}}^{\boldsymbol{T}}+\boldsymbol{c}_{2}^{\boldsymbol{T}}\right) & A_{q}+\kappa \boldsymbol{b}_{2} \boldsymbol{c}_{\boldsymbol{q}}^{\boldsymbol{T}} & -\boldsymbol{b}_{2} \gamma \\
0 & \kappa\left(\boldsymbol{c}_{\boldsymbol{q}}^{\boldsymbol{T}}+\boldsymbol{c}_{2}^{\boldsymbol{T}}\right) & \kappa \boldsymbol{c}_{\boldsymbol{q}}^{\boldsymbol{T}} & 0
\end{array}\right]\left[\begin{array}{c}
z_{1} \\
z_{2} \\
\chi \\
\eta
\end{array}\right]+\left[\begin{array}{c}
0 \\
-\boldsymbol{b}_{2} \\
-\boldsymbol{b}_{2} \\
v
\end{array}\right] \psi+\left[\begin{array}{c}
R_{1} \\
R_{2} \\
R_{2} \\
0
\end{array}\right] \omega .} \\
& {\left[\begin{array}{c}
\dot{z}_{1} \\
\dot{\zeta} \\
\dot{\chi} \\
\dot{z}_{2}
\end{array}\right]=\left[\begin{array}{cccc}
A_{11} & 0 & A_{12} & 0 \\
\bar{b}_{2}^{T} A_{21} & -\gamma & \bar{b}_{2}^{T}\left(A_{22}+\gamma I\right) & 0 \\
A_{21} & -\boldsymbol{b}_{2} \gamma & A_{22}+I_{0}-\kappa \boldsymbol{b}_{2}\left(\boldsymbol{c}_{\boldsymbol{q}}^{\boldsymbol{T}}+\boldsymbol{c}_{2}^{\boldsymbol{T}}\right)+\gamma \boldsymbol{b}_{2} \bar{b}_{2}^{T} & A_{q}+\kappa \boldsymbol{b}_{2} \boldsymbol{c}_{\boldsymbol{q}}^{\boldsymbol{T}} \\
A_{21} & -\boldsymbol{b}_{2} \gamma & A_{22}-\kappa \boldsymbol{b}_{2}\left(\boldsymbol{c}_{\boldsymbol{q}}^{\boldsymbol{T}}+\boldsymbol{c}_{2}^{\boldsymbol{T}}\right)+\gamma \boldsymbol{b}_{\mathbf{2}} \bar{b}_{2}^{T} & \kappa \boldsymbol{b}_{2} \boldsymbol{c}_{\boldsymbol{q}}^{\boldsymbol{T}}
\end{array}\right]\left[\begin{array}{c}
z_{1} \\
\zeta \\
\chi \\
z_{2}
\end{array}\right]+\left[\begin{array}{c}
0 \\
(v-1) \\
-\boldsymbol{b}_{2} \\
-\boldsymbol{b}_{2}
\end{array}\right] \psi+\left[\begin{array}{c}
R_{1} \\
\bar{b}_{2}^{T} R_{2} \\
R_{2} \\
R_{2}
\end{array}\right] \omega}
\end{aligned}
$$

As noted above, the matrix $A_{11}$ is Hurwitz due to Assumption 1. The matrix

$$
A_{1}=\left[\begin{array}{cc}
A_{11} & 0 \\
\bar{b}_{2}^{T} A_{21} & -\gamma
\end{array}\right]
$$

is Hurwitz due to the choice of the parameter $\gamma>0$.

There exists a number $\kappa_{0}$ such that for $\forall \kappa \geq \kappa_{0}$ the matrix

$$
A_{2}=\left[\begin{array}{ccc}
A_{11} & 0 & A_{12} \\
\bar{b}_{2}^{T} A_{21} & -\gamma & \bar{b}_{2}^{T}\left(A_{22}+\gamma I\right) \\
A_{21} & -b_{2} \gamma & A_{22}-\kappa b_{2}\left(c_{q}^{T}+c_{2}^{T}\right)+\gamma b_{2} \bar{b}_{2}^{T}
\end{array}\right]
$$

is Hurwitz, since only the element $(3,3)$ depends on the parameter $\kappa$, which can be set to be sufficiently large.

The overall state matrix of the closed-loop system (16) can be forced to be Hurwitz due to the structure of the matrix $A_{q}$ and sufficiently large value of the parameter $\sigma$, which is included only in the block $(4,4)$.

Statement 1: Consider the plant model given at statespace (7), (8) and the control law (9)-(12). Hurwitzness of the state matrix of the resultant closed-loop system (16) follows from Hurwitzness of all its diagonal blocks, which is forced by the parameters $\gamma, \kappa, \sigma$.

\section{Stability analisys of closed-loop system}

Consider the case without taking into account the external disturbances, i.e. $\omega=0$. Denote

$$
F=\left[\begin{array}{ccc}
A-\kappa \boldsymbol{b} \boldsymbol{c}^{T} & -\kappa \boldsymbol{b} \boldsymbol{c}_{q}^{T} & -\gamma \boldsymbol{b} \\
\boldsymbol{b}_{q} \boldsymbol{c}^{T} & A_{q} & 0 \\
\kappa \boldsymbol{c}^{T} & \kappa \boldsymbol{c}_{q}^{T} & 0
\end{array}\right], \boldsymbol{g}=\left[\begin{array}{c}
-\boldsymbol{b} \\
0 \\
v
\end{array}\right], \boldsymbol{h}=\left[\begin{array}{c}
-\kappa \boldsymbol{c} \\
-\kappa c_{q} \\
-\gamma
\end{array}\right], \varsigma=\left[\begin{array}{l}
x \\
\xi \\
\eta
\end{array}\right],
$$

Represent the closed-loop system (16) using notations (17) as follows

$$
\begin{gathered}
\dot{\boldsymbol{s}}=F \boldsymbol{x}+\boldsymbol{g} \psi(v), \\
v=\boldsymbol{h}^{T} \boldsymbol{s} .
\end{gathered}
$$

According to the Popov criterion [22], the closedloop system consisting of the linear part (18)-(19) with Hurwitz state matrix $F$ and the memoryless nonlinear function $\psi(v)$ is absolutely stable for all initial conditions if there exists a constant $\bar{\omega} \geq 0$ such that $\left(1+\lambda_{i} \bar{\omega}\right) \neq 0$ for each eigenvalue $\lambda_{i}$ of the matrix $F$ and the transfer function $W(s)=1+(1+s \bar{\omega}) W_{l}(s)$ is strictly positive real, where $W_{l}(s)$ is the transfer function of the system (18)-(19).

The transfer function $W_{l}(s)$ can be derived as follows

$$
\begin{aligned}
& W_{l}(s)=\boldsymbol{h}^{\boldsymbol{T}}(s I-F)^{-1} \boldsymbol{g} \\
& =\left[\begin{array}{lll}
-\kappa \boldsymbol{c}^{\boldsymbol{T}} & -\kappa \boldsymbol{c}_{\boldsymbol{q}}^{\boldsymbol{T}} & -\gamma
\end{array}\right] \mathcal{F}\left[\begin{array}{c}
-b \\
0 \\
v
\end{array}\right],
\end{aligned}
$$

where

$$
\mathcal{F}=(s I-F)^{-1}=\left[\begin{array}{ccc}
s I-A+\kappa \boldsymbol{b} \boldsymbol{c}^{\boldsymbol{T}} & \kappa \boldsymbol{b} \boldsymbol{c}_{\boldsymbol{q}}^{\boldsymbol{T}} & \gamma \boldsymbol{b} \\
-\boldsymbol{b}_{\boldsymbol{q}} \boldsymbol{c}^{\boldsymbol{T}} & s I-A_{q} & 0 \\
-\kappa \boldsymbol{c}^{\boldsymbol{T}} & -\kappa \boldsymbol{c}_{q}^{T} & s
\end{array}\right]^{-1}
$$

Represent the matrix $\mathcal{F}$ in the block form

$$
\mathcal{F}=\overline{\mathcal{F}}^{-1}=\left[\begin{array}{ll}
\overline{\mathcal{F}}_{11} & \overline{\mathcal{F}}_{12} \\
\overline{\mathcal{F}}_{21} & \overline{\mathcal{F}}_{22}
\end{array}\right]=\left[\begin{array}{ll}
\mathcal{F}_{11} & \mathcal{F}_{12} \\
\mathcal{F}_{21} & \mathcal{F}_{22}
\end{array}\right]
$$

where

$$
\begin{gathered}
\overline{\mathcal{F}}_{11}=s I-A+\kappa \boldsymbol{b} \boldsymbol{c}^{T}, \quad \overline{\mathcal{F}}_{12}=\left[\begin{array}{ll}
\kappa \boldsymbol{b} \boldsymbol{c}_{\boldsymbol{q}}^{\boldsymbol{T}} & \gamma \boldsymbol{b}
\end{array}\right], \\
\overline{\mathcal{F}}_{21}=\left[\begin{array}{c}
-\boldsymbol{b}_{\boldsymbol{q}} \boldsymbol{c}^{\boldsymbol{T}} \\
-\kappa \boldsymbol{c}^{\boldsymbol{T}}
\end{array}\right], \quad \overline{\mathcal{F}}_{22}=\left[\begin{array}{cc}
s I-A_{q} & 0 \\
-\kappa \boldsymbol{c}_{q}^{\boldsymbol{T}} & s
\end{array}\right],
\end{gathered}
$$

and use the Frobenius formula for block matrices inversion.

Calculate the inverse matrix of the block $\overline{\mathcal{F}}_{22}$

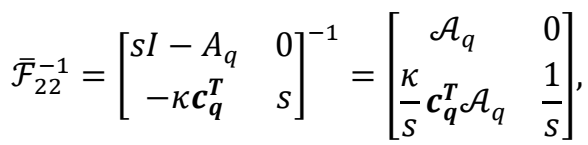


where $\mathcal{A}_{q}=\left(s I-A_{q}\right)^{-1}$.

Calculate the block $\mathcal{F}_{11}$

$$
\begin{aligned}
\mathcal{F}_{11}=\left(\overline{\mathcal{F}}_{11}-\overline{\mathcal{F}}_{12} \overline{\mathcal{F}}_{22}^{-1} \overline{\mathcal{F}}_{21}\right)^{-1} & \\
& =\left(s I-A+\boldsymbol{b} \frac{\kappa b_{q}(s)(s+\gamma)}{s a_{q}(s)} \boldsymbol{c}^{\boldsymbol{T}}\right)^{-1},
\end{aligned}
$$

where

$$
\frac{b_{q}(s)}{a_{q}(s)}=\boldsymbol{c}_{\boldsymbol{q}}^{T} \mathcal{A}_{q}(s) \boldsymbol{b}_{\boldsymbol{q}}+1 .
$$

Remark 1: Note that, Hurwitzness of the numerator and denominator of the transfer function (22) can be achieved by choosing matrix $A_{q}$ and vectors $\boldsymbol{b}_{q}$ and $\boldsymbol{c}_{\boldsymbol{q}}$.

Calculate the block $\mathcal{F}_{12}$

$$
\begin{aligned}
& \mathcal{F}_{12}=-\mathcal{F}_{11} \overline{\mathcal{F}}_{12} \overline{\mathcal{F}}_{22}^{-1}
\end{aligned}
$$

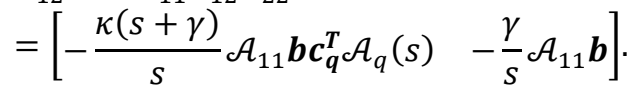

Calculate the block $\mathcal{F}_{21}$

$$
\begin{aligned}
\mathcal{F}_{21}=-\overline{\mathcal{F}}_{22}^{-1} \overline{\mathcal{F}}_{21} \mathcal{F}_{11}=\left[\begin{array}{c}
\mathcal{A}_{q}(s) \boldsymbol{b}_{q} \boldsymbol{c}^{\boldsymbol{T}} \\
\kappa \\
\frac{\kappa}{s}\left(\boldsymbol{c}_{q}^{T} \mathcal{A}_{q}(s) \boldsymbol{b}_{q}+1\right) \boldsymbol{c}^{T}
\end{array}\right] \mathcal{F}_{11} \\
=\left[\begin{array}{c}
\mathcal{A}_{q}(s) \boldsymbol{b}_{q} \boldsymbol{c}^{T} \mathcal{F}_{11} \\
\frac{\kappa b_{q}(s)}{s a_{q}(s)} \boldsymbol{c}^{T} \mathcal{F}_{11}
\end{array}\right] .
\end{aligned}
$$

Calculate the block $\mathcal{F}_{22}$

$$
\begin{gathered}
\mathcal{F}_{22}=\overline{\mathcal{F}}_{22}^{-1}-\mathcal{F}_{21} \overline{\mathcal{F}}_{12} \overline{\mathcal{F}}_{22}^{-1}= \\
{\left[\begin{array}{cc}
\left(1-\frac{\kappa(s+\gamma)}{s} \mathcal{A}_{q}(s) \boldsymbol{b}_{q} \frac{\beta(s)}{\alpha(s)} \boldsymbol{c}_{q}^{T}\right) \mathcal{A}_{q}(s) & -\frac{\gamma}{s} \mathcal{A}_{q}(s) \boldsymbol{b}_{q} \frac{\beta(s)}{\alpha(s)} \\
\left.\left(1-\frac{\kappa(s+\gamma) b_{q}(s)}{s a_{q}(s)} \frac{\beta(s)}{\alpha_{q}(s)}\right)\right)_{s} \boldsymbol{c}_{q} \boldsymbol{c}_{\boldsymbol{q}}^{T} \mathcal{A}_{q}(s) & \left(1-\frac{\gamma \kappa b_{q}(s)}{s a_{q}(s)} \frac{\beta(s)}{\alpha(s)}\right)
\end{array}\right)}
\end{gathered}
$$

where

$$
\begin{aligned}
& \frac{\beta(s)}{\alpha(s)}=\boldsymbol{c}^{\boldsymbol{T}} \mathcal{F}_{11} \boldsymbol{b} \\
& =\frac{b(s) s a_{q}(s)}{s a_{q}(s) a(s)+\kappa b_{q}(s)(s+\gamma) b(s)} .
\end{aligned}
$$

Substitute derived expressions of the blocks $\mathcal{F}_{11}, \mathcal{F}_{12}, \mathcal{F}_{21}$ and $\mathcal{F}_{22}$ into the matrix $\mathcal{F}$ (for the sake of brevity the middle column is omitted)

$$
\mathcal{F}=\left[\begin{array}{ccc}
\mathcal{F}_{11} & * & -\frac{\gamma}{s} \mathcal{F}_{11} \boldsymbol{b} \\
\mathcal{A}_{q}(s) \boldsymbol{b}_{q} \boldsymbol{c}^{\boldsymbol{T}} \mathcal{F}_{11} & * & -\frac{\gamma}{s} \mathcal{A}_{q}(s) \boldsymbol{b}_{\boldsymbol{q}} \frac{\beta(s)}{\alpha(s)} \\
\frac{\kappa b_{q}(s)}{s a_{q}(s)} \boldsymbol{c}^{\boldsymbol{T}} \mathcal{F}_{11} & * & \left(1-\frac{\gamma \kappa b_{q}(s)}{s a_{q}(s)} \frac{\beta(s)}{\alpha(s)}\right) \frac{1}{s}
\end{array}\right] .
$$

Substitute the matrix $\mathcal{F}$ in (21) and obtain

$$
W_{l}(s)=\beta_{\kappa}(s)-\frac{\gamma v a_{q}(s) a(s)}{s a_{q}(s) a(s)}+\beta_{\kappa}(s),
$$

where

$$
\beta_{\kappa}(s)=\kappa b_{q}(s)(s+\gamma) b(s) .
$$

Set $\bar{\omega}=0$, then the sufficient condition of the absolute stability of the system is strictly positive realness of the transfer function $W(s)=1+W_{l}(s)$. Calculate $W(s)$

$$
\begin{aligned}
& W(s)=W_{l}(s)+1=2 \beta_{\kappa}(s)+\frac{(s-v \gamma) a_{q}(s) a(s)}{s a_{q}(s) a(s)+\beta_{\kappa}(s)} \\
& =\frac{2 \kappa b_{q}(s)(s+\gamma) b(s)+(s-\gamma v) a_{q}(s) a(s)}{s a_{q}(s) a(s)+\kappa b_{q}(s)(s+\gamma) b(s)} .
\end{aligned}
$$

It easy to show, that there exists a number $\kappa_{0}$ such that for $\kappa \geq \kappa_{0}$ both the numerator and denominator of the transfer function $W(s)$ are Hurwitz. The relative degree of $W(s)$ is zero. As a consequence, strictly positive realness follows, i.e. $\operatorname{Re} W(j \omega)>0, \forall \omega \in$ $[-\infty, \infty]$ or equivalently $\operatorname{Re} W_{l}(j \omega)>1, \forall \omega \in[-\infty, \infty]$ and the absolute stability of the system (18), (19) follows in accordance to the Popov criterion [22].

Consider the case taking into account the external disturbances, i.e. $\omega \neq 0$, and analyze the steady error. Obviously, at steady state the relations $v=\operatorname{sat}(v)$ and $\psi(v)=v-\operatorname{sat}(v)=0$ hold.

In order to determine steady state behavior use the Sylvester equation applied to the model (13) and focus on the fourth row

$$
\Sigma S=\kappa\left(\boldsymbol{c}_{2}^{T} \Pi_{2}+\boldsymbol{c}_{\boldsymbol{q}}^{T} \Pi_{\xi}\right),
$$

which shows that at steady state

$$
c_{2}^{T} \Pi_{2}+c_{q}^{T} \Pi_{\xi}=0 .
$$

Find the relation between $\boldsymbol{c}_{2}^{T} \Pi_{2}$ and $\boldsymbol{c}_{\boldsymbol{q}}^{T} \Pi_{\xi}$. Consider the auxiliary variable $z_{0}$

$$
z_{0}=y+c_{q}^{T} \xi,
$$

taking into account (24), the steady state value of which is zero.

From (10) follows

$$
\xi(s)=\left(s I-A_{q}\right)^{-1}\left(\boldsymbol{b}_{\boldsymbol{q}} y(s)+\xi(0)\right),
$$

where $\xi(0)$ is a vector of initial conditions. Rewrite (25)

$$
z_{0}(s)=\left(\boldsymbol{c}_{\boldsymbol{q}}^{\boldsymbol{T}}\left(s I-A_{q}\right)^{-1} \boldsymbol{b}_{\boldsymbol{q}}+1\right) y(s)+\epsilon(s),
$$

where $\quad \epsilon(s)=\boldsymbol{c}_{\boldsymbol{q}}^{T}\left(s I-A_{q}\right)^{-1}\left(\xi_{1}(0)+\xi_{2}(0)\right)$ corresponds to the exponentially decaying function $\epsilon(t)$. If $\boldsymbol{c}_{\boldsymbol{q}}^{\boldsymbol{T}}$ is chosen so that the numerator of the transfer function $\left(\boldsymbol{c}_{\boldsymbol{q}}^{\boldsymbol{T}}\left(s I-A_{q}\right)^{-1} \boldsymbol{b}_{\boldsymbol{q}}+1\right)$ is Hurwitz and the relative degree is zero, then from

$$
y(s)=\left(\boldsymbol{c}_{\boldsymbol{q}}^{\boldsymbol{T}}\left(s I-A_{q}\right)^{-1} \boldsymbol{b}_{\boldsymbol{q}}+1\right)^{-1}\left(z_{0}(s)-\epsilon(s)\right)
$$

find that the steady-state error of y as well as $\boldsymbol{c}_{\boldsymbol{q}}^{T} \xi$ converges to zero. 


\section{Experimental verification}

The practical verification of this research has been carried out using the robotic boat shown in Figure 1. It is intended to test DP systems of marince CPS under laboratory conditions.

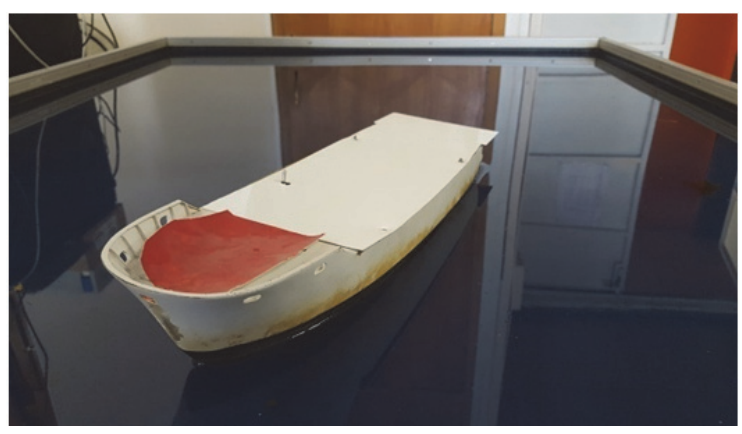

Fig. 1. Robotic boat.

The boat is equipped with the main engine, bow and stern tunnel thrusters and heading servo drive (Fig. 2). Dimensions of the boat are $(0.432 \times 0.096 \times 0.052) \mathrm{m}$. Its workspace is represented by the specific basin made of plywood sheets with dimensions $(1.50 \times 1.10 \times 0.1) \mathrm{m}$. The computer vision system emulating satelite navigation systems is based on the RGB video signal provided by the digital camera placed above the basin. The control signals transmitted via radio channel saturate at the rates $[-127 ; 127]$ due to the pulse-width modulation technique.

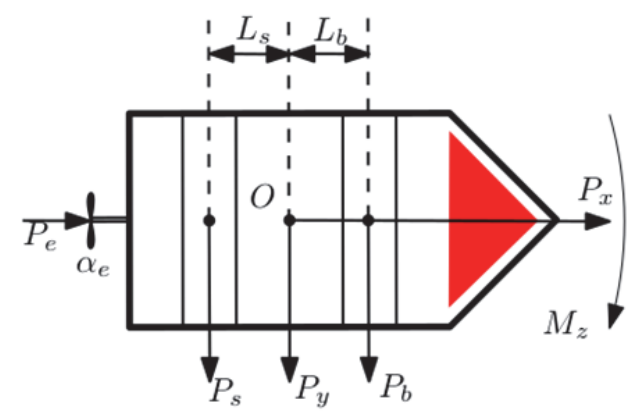

Fig. 2. Actuator configuration of the robotic boat.

The particular form of the control law is (9)-(12) applied to the boat model with the relative degree being equal to 2 is

$$
A_{q}=\left[\begin{array}{cc}
-q_{2}^{\prime} \sigma & 1 \\
-q_{1}^{\prime} \sigma^{2} & 0
\end{array}\right], \quad \boldsymbol{b}_{\boldsymbol{q}}=\left[\begin{array}{c}
q_{2}^{\prime} \sigma \\
q_{1}^{\prime} \sigma^{2}
\end{array}\right], \quad \boldsymbol{c}_{\boldsymbol{q}}=\left[\begin{array}{l}
q_{1} \\
q_{2}
\end{array}\right]
$$

Three experiments have been carried out. The first one focuses on the simple robust output control with the internal model (11) deactivated ( $\operatorname{set} \gamma=0$ ). The second one is the robuts output control with the integral term and excluding the anti-windup scheme (12) (set $\gamma \neq$ $0, v=0)$. The third one is robuts output control with the integral term and anti-windup scheme, which is the suggested algorithm (set $\gamma \neq 0, v=1)$.

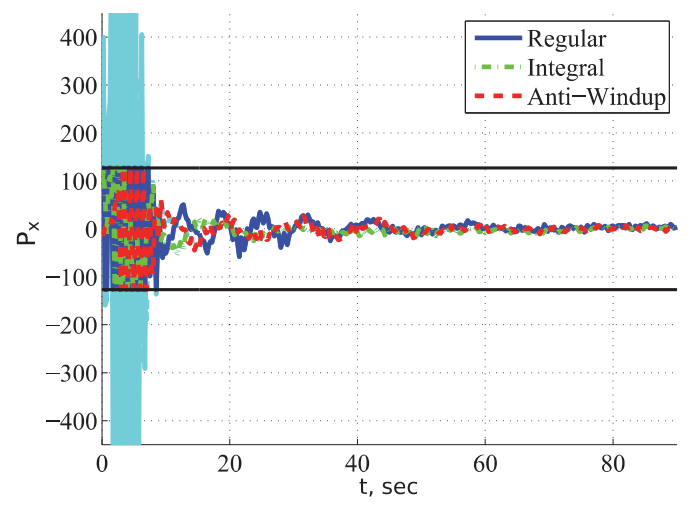

Fig. 3. Plots of the saturated control $x$-axis.

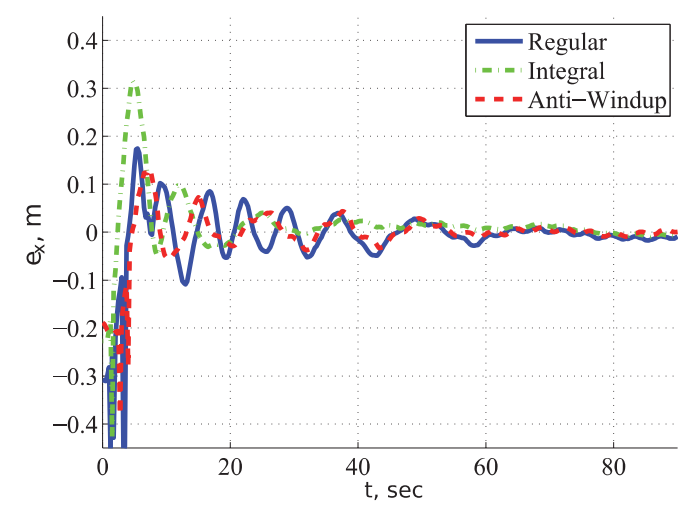

Fig. 4. Plots of the error signals along $x$-axis.

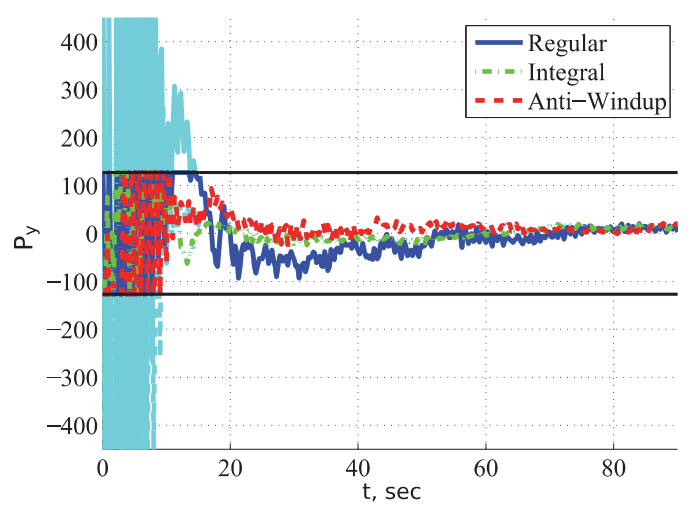

Fig. 5. Plots of the saturated control along $y$-axis.

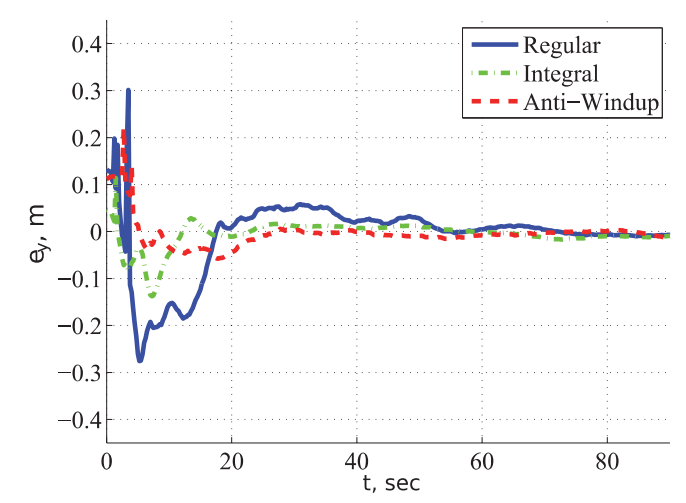

Fig. 6. Plots of the error signals along $y$-axis. 


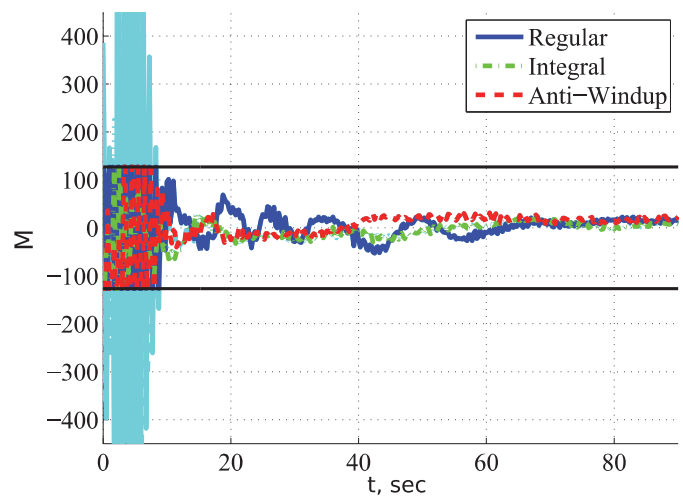

Fig. 7. Plots of the saturated control along $\psi$-axis.

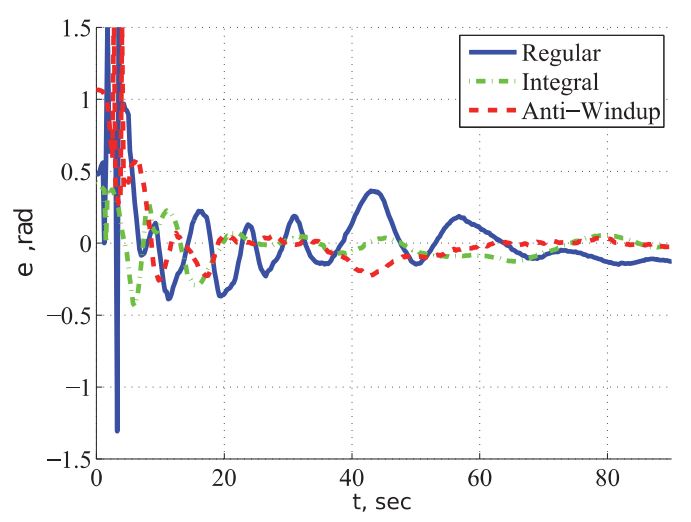

Fig. 8. Plots of the error signals along $\psi$-axis.

The experimental results are given in Figures 3-8. Cyan curves illustrate the control signals generated by the nominal controller without saturation. Blue curves are assigned to the simplest controller with the integral term deactivated. Green curves correspond to the controller augmeted with the integral term. Red curves refer to the controller augmented with the itegral term and anti-windup scheme.

The experimental data shows that the first controller leads to the slight steady-state error. It is compensated by means of the integral term which accumulates the error value and increase the control signal. In this case integral windup occurs and leads to the overshoot increase. This effect can be fixed using the anti-windup approach.

\section{Conclusions}

This research focuses on the design the robust output control algorithm with anti-windup compensation for DP systems. The theoretical background is based on the consecutive compensator approach, which uses the highgain principle to suppress plant uncertainties. The paper provides control design strategy, strict stability analysis and experimental verification by means of the robotic boat setup.

This article is supported by the Russian Federation President Grant 14.Y31.16.9281-HШ, the Government of the Russian Federation (grant 074-U01), the Ministry of Education and Science of the Russian Federation (goszadanie no 2.8878.2017/8.9, project 14.Z50.31.0031) and Russian Foundation for Basic Research (project 17-58-53129).

\section{References}

1. H.A. Fertik, C.W. Ross, ISA transactions, 6, 317 (1967)

2. S. Galeani, S. Tarbouriech, M. Turner, L. Zaccarian, ECC 2009, 306-323 (2014)

3. G. Grimm, J. Hatfield, I. Postlethwaite, A.R. Teel, A.C. Turner, L. Zaccarian, IEEE Trans. Autom. Control, 48 (9), 1059-1525 (2003)

4. E.F. Mulder, M.V. Kothare, M. Morari, Automatica, 37, 1407-1416 (2001)

5. M. Hussain, N. Us Saqib, M. Rehan, ICET 2016, 7813237 (2016)

6. T. Hu, J. A.R. Teel, L. Zaccarian, Automatica, 44, 512-519 (2008)

7. J.M. Gomes Da Silva, S. Tarbouriech, R. Reginatto, 2002 IEEE Int. Symp. Comp. Aided Control Systems Design Proc., 2, 1106-1111 (2002)

8. M.V. Kothare, M. Morari, 1997 ECC, 2156-2161 (1997)

9. A. Zheng, M. V. Kothare, M. Morari, Int. J. Control, 60, 1015-1024 (1994)

10. Z. Liu, J. Wang, Q. Jin, L. Qi, B. Jiang, DDCLS 2017, 512-517 (2017)

11. W. Wu, $201150^{\text {th }}$ IEEE CDC-ECC, 4783-4788 (2011)

12. J.M. Gomes Da Silva, R. Reginatto, S. Tarbouriech, Controle and Automacao, 15 (1), 3-9 (2004)

13. A.A. Bobtsov, N.A. Nikolaev, Autom. Remote Control, 66, 108-118 (2005)

14. A.A. Bobtsov, Automatica, 41, 2177-2180 (2005)

15. A. Fradkov, Eur. J. Control, 9, 577-586 (2003)

16. A. Pyrkin, A. Isidori, 50, 5498 - 5503 (2017)

17. T. Fossen, Marine control systems: Guidance, Navigation, and Control of Ships, Rigs and Underwater Vehicles (2002)

18. O. Borisov, V. Gromov, A. Pyrkin, A. Bobtsov, I. Petranevsky, A. Klyunin, MMAR 2016, 13-18 (2016)

19. O. Borisov, V. Gromov, A. Pyrkin, A. Bobtsov, N. Nikolaev, IFAC-PapersOnLine, 49, 256-261 (2016)

20. O. Borisov, A. Bobtsov, A. Pyrkin, V. Gromov, AIP Conference Proceedings, 1798 (2017)

21. O. Borisov, V. Gromov, A. Pyrkin, A. Bobtsov, N. Nikolaev, IFAC-PapersOnLine, 49, 287-292 (2016).

22. S. Tomashevich, O. Borisov, V. Gromov, A. Pyrkin, A. Bobtsov, MED 2017, 1029-1034 (2017).

23. H. K. Khalil, Nonlinear systems (2002). 